\title{
Separating sets by Darboux-like functions
}

\author{
by
}

Aleksander Maliszewski (Bydgoszcz)

\begin{abstract}
We consider the following problem: Characterize the pairs $\langle A, B\rangle$ of subsets of $\mathbb{R}$ which can be separated by a function from a given class, i.e., for which there exists a function $f$ from that class such that $f=0$ on $A$ and $f=1$ on $B$ (the classical separation property) or $f<0$ on $A$ and $f>0$ on $B$ (a new separation property).
\end{abstract}

1. Introduction. The classical Urysohn Lemma states that if $(X, \mathcal{T})$ is a $T_{4}$-space and the sets $A_{0}, A_{1} \subset X$ are disjoint and closed, then there is a continuous function $f: X \rightarrow \mathbb{R}$ such that $f=0$ on $A_{0}$ and $f=1$ on $A_{1}$, and if moreover $A_{0}$ and $A_{1}$ are $G_{\delta^{-}}$sets, then $0<f<1$ on $X \backslash\left(A_{0} \cup A_{1}\right)$. In the first part of the paper we examine when, given two sets $A_{0}, A_{1} \subset \mathbb{R}$, we can find a Darboux (or functionally connected, or connected, or almost continuous) function $f$ such that $f=0$ on $A_{0}$ and $f=1$ on $A_{1}$. It turns out that the necessary-and-sufficient condition is the same in all the cases we consider (Corollaries 3.2 and 3.3).

In the second part we study a similar problem. Namely we ask when, given two sets $A^{-}, A^{+} \subset \mathbb{R}$, we can find a Darboux (or functionally connected, or connected, or almost continuous) function $f$ such that $f<0$ on $A^{-}$and $f>0$ on $A^{+}$. It is surprising that we get three essentially different necessary-and-sufficient conditions. (See examples in Section 5.)

2. Preliminaries. The letters $\mathbb{R}$ and $\mathbb{N}$ denote the real line and the set of positive integers, respectively. The word interval means a nondegenerate interval; $[a, b]$ denotes the closed interval with end points $a$ and $b$ also in case $a>b$. The word function denotes a mapping from $\mathbb{R}$ into $\mathbb{R}$ unless otherwise explicitly stated. For each $A \subset \mathbb{R}$ we denote by $\operatorname{Int} A, \operatorname{cl} A$, and card $A$ the

2000 Mathematics Subject Classification: Primary 26A21, 54C30; Secondary 26A15, $54 \mathrm{C} 08$.

Key words and phrases: Darboux property, connectivity, almost continuity.

Supported by Bydgoszcz Academy. 
interior, closure, and cardinality of $A$, respectively. We write $\mathfrak{c}=$ card $\mathbb{R}$. If $K \subset \mathbb{R}^{2}$, then we let dom $K$ be the $x$-projection of $K$.

Let $A \subset \mathbb{R}$. By $A^{\star}$ we denote the set of all $x \in A$ which are bilateral c-limit points of $A$, i.e.,

$$
A^{\star}=\{x \in A: \operatorname{card}(A \cap[x, t])=\mathfrak{c} \text { for each } t \neq x\} .
$$

We say that a set $A \subset \mathbb{R}$ is bilaterally $\mathfrak{c}$-dense in itself if $A=A^{\star}$. Recall that for each $A \subset \mathbb{R}$, we have $\operatorname{card}\left(A \backslash A^{\star}\right)<\mathfrak{c}$. (See, e.g., [1, Lemma 4, p. 285].) So, $\left(A^{\star}\right)^{\star}=A^{\star}$.

Let $f: \mathbb{R} \rightarrow \mathbb{R}$. For every $y \in \mathbb{R}$ let $[f<y]=\{x \in \mathbb{R}: f(x)<y\}$. Similarly we define the symbols $[f \leq y],[f>y]$, etc. For each $x \in \mathbb{R}$ and $\delta>0$ we set

$$
\mathrm{R}_{f}(x, \delta)=(x-\delta, x+\delta) \times(f(x)-\delta, f(x)+\delta) .
$$

Usually we will write $\mathrm{R}(x, \delta)$ instead of $\mathrm{R}_{f}(x, \delta)$, if it does not lead to misunderstanding.

Let $f: \mathbb{R} \rightarrow \mathbb{R}$. We say that $f$ is a Darboux function if it has the intermediate value property. We say that $f$ is connected if it is a connected subset of $\mathbb{R}^{2}$. (We make no distinction between a function and its graph.) We say that $f$ is functionally connected if $g \cap f \neq \emptyset$ whenever $g:[\alpha, \beta] \rightarrow \mathbb{R}$ is a continuous function such that $(f(\alpha)-g(\alpha))(f(\beta)-g(\beta))<0$. We say that $f$ is almost continuous in the sense of Stallings [6] if for every open set $V \subset \mathbb{R}^{2}$ containing $f$, there is a continuous function $h: \mathbb{R} \rightarrow \mathbb{R}$ with $h \subset V$. One can easily see that

$$
\begin{aligned}
\text { almost continuity } & \Rightarrow \text { connectivity } \\
& \Rightarrow \text { functional connectivity } \Rightarrow \text { Darboux property. }
\end{aligned}
$$

(See also [6].) Though the above implications cannot be reversed, the algebraic properties of the corresponding classes of functions are very similar. See, e.g., [5] or [2] for details.

\section{Classical separation property}

Proposition 3.1. Assume that the sets $A_{0}, A_{1} \subset \mathbb{R}$ satisfy the following conditions:

$$
\begin{aligned}
& \mathbb{R} \backslash\left(A_{0} \cup A_{1}\right)=\left(\mathbb{R} \backslash\left(A_{0} \cup A_{1}\right)\right)^{\star} ; \\
& \text { for all } \alpha \in A_{0} \text { and } \beta \in A_{1} \text {, we have } \operatorname{card}\left([\alpha, \beta] \backslash\left(A_{0} \cup A_{1}\right)\right)=\mathfrak{c} \text {. }
\end{aligned}
$$

Then there is an almost continuous function $f$ such that

$$
A_{0}=[f=0], \quad A_{1}=[f=1] .
$$

Proof. Clearly we may assume that $A_{0} \neq \mathbb{R} \neq A_{1}$. Let $X=\mathbb{R} \times(0,1)$ and let $\left\{K_{\xi}: \xi<\mathfrak{c}\right\}$ be an enumeration of all closed sets $K \subset \mathbb{R}^{2}$ such 
that $\operatorname{card}\left(\operatorname{dom}(K \cap X) \backslash\left(A_{0} \cup A_{1}\right)\right)=\mathfrak{c}$. For each $\xi<\mathfrak{c}$ choose a point $\left\langle x_{\xi}, y_{\xi}\right\rangle \in K_{\xi} \cap X$ such that $x_{\xi} \notin A_{0} \cup A_{1} \cup\left\{x_{\zeta}: \zeta<\xi\right\}$. Define

$$
f(x)= \begin{cases}0 & \text { if } x \in A_{0}, \\ y_{\xi} & \text { if } x=x_{\xi}, \xi<\mathfrak{c} \\ 1 & \text { if } x \in A_{1} \\ 1 / 2 & \text { otherwise. }\end{cases}
$$

Evidently $f$ satisfies (3.3). We will show that $f$ is almost continuous.

Take an arbitrary open set $V \supset f$. Clearly it suffices to show that for all $a<b$ there is a continuous function $h:[a, b] \rightarrow \mathbb{R}$ contained in $V$ such that $h=f$ on $\{a, b\}$.

Fix $a<b$. Let $S$ be the set of all $s \in[a, b]$ for which there is a continuous function $h:[s, b] \rightarrow \mathbb{R}$ contained in $V$ such that $h=f$ on $\{s, b\}$. Then $b \in S$, so we can define $x_{0}=\inf S$. First we will prove that $x_{0} \in S$.

Indeed, suppose that this is not the case. Then $x_{0}<b$. Let $\delta>0$ be such that $\mathrm{R}\left(x_{0}, \delta\right) \subset V$. (Recall that $V$ is open and $f \subset V$.) Fix an $x \in S \cap\left(x_{0}, x_{0}+\delta\right)$. Set

$$
x_{1}=\inf \left\{z \in\left(x_{0}, x\right]: f \text { is constant on }[z, x]\right\} .
$$

Notice that by construction and (3.1), $f\left(x_{1}\right)=f(x)$. So, $x_{1} \in S$ and $x_{1}>x_{0}$. Using the fact that $\mathbb{R}^{2} \backslash V$ is closed and $f \subset V$, we obtain $\operatorname{card}\left\{z \notin A_{0} \cup A_{1}:\{z\} \times(0,1) \not \subset V\right\}=\operatorname{card}\left(\operatorname{dom}(X \backslash V) \backslash\left(A_{0} \cup A_{1}\right)\right)<\mathfrak{c}$. Let $\tau>0$ be such that $x_{0}<x_{1}-\tau$ and $\mathrm{R}\left(x_{1}, \tau\right) \subset V$. Observe that by (3.2), $x_{1} \in \operatorname{cl}\left((-\infty, x) \backslash\left(A_{0} \cup A_{1}\right)\right)$. So by (3.1), there is an $x_{2} \in\left(x_{1}-\tau, x_{1}\right)$ such that $\left\{x_{2}\right\} \times(0,1) \subset V$.

Pick $y_{0} \in\left(f\left(x_{0}\right)-\delta, f\left(x_{0}\right)+\delta\right) \cap(0,1)$ and $y_{1} \in\left(f\left(x_{1}\right)-\tau, f\left(x_{1}\right)+\tau\right) \cap$ $(0,1)$. Using the compactness of the set $\left\{x_{2}\right\} \times\left[y_{0}, y_{1}\right] \subset V$, we can find an $\eta>0$ such that $x_{2}+\eta<x_{1}$ and $\left(x_{2}-\eta, x_{2}+\eta\right) \times\left[y_{0}, y_{1}\right] \subset V$. Let $h_{0}$ correspond to $x_{1} \in S$. Extend $h_{0}$ to $h:\left[x_{0}, b\right] \rightarrow \mathbb{R}$ by connecting the following pairs of points by straight line segments: $\left\langle x_{0}, f\left(x_{0}\right)\right\rangle$ with $\left\langle x_{2}, y_{0}\right\rangle,\left\langle x_{2}, y_{0}\right\rangle$ with $\left\langle x_{2}+\eta, y_{1}\right\rangle$, and $\left\langle x_{2}+\eta, y_{1}\right\rangle$ with $\left\langle x_{1}, f\left(x_{1}\right)\right\rangle$. Clearly the function $h$ proves $x_{0} \in S$, contrary to assumption.

Now suppose that $x_{0}>a$. Let $\delta>0$ be such that $a<x_{0}-\delta$ and $\mathrm{R}\left(x_{0}, \delta\right) \subset V$. By (3.1), there is an $x \in\left(x_{0}-\delta, x_{0}\right)$ with $\left|f(x)-f\left(x_{0}\right)\right|<\delta$. Let $h_{0}$ correspond to $x_{0} \in S$. Extend $h_{0}$ to $h:[x, b] \rightarrow \mathbb{R}$ by connecting $\langle x, f(x)\rangle$ with $\left\langle x_{0}, f\left(x_{0}\right)\right\rangle$ by a straight line segment. Clearly this function proves $x \in S$. But $x<x_{0}=\inf S$, an impossibility.

We have proved that $a \in S$. So, there is a continuous function $h:[a, b]$ $\rightarrow \mathbb{R}$ contained in $V$ such that $h=f$ on $\{a, b\}$.

Corollary 3.2. Let $A_{0}, A_{1} \subset \mathbb{R}$. The following are equivalent:

(i) the sets $A_{0}$ and $A_{1}$ satisfy conditions (3.1) and (3.2), 
(ii) there is an almost continuous function $f$ such that (3.3) holds,

(iii) there is a connected function $f$ such that (3.3) holds,

(iv) there is a functionally connected function $f$ such that (3.3) holds,

(v) there is a Darboux function $f$ such that (3.3) holds.

Proof. The implication (i) $\Rightarrow$ (ii) follows by Proposition 3.1.

The implications (ii) $\Rightarrow$ (iii) $\Rightarrow$ (iv) $\Rightarrow(\mathrm{v})$ are immediate.

$(\mathrm{v}) \Rightarrow(\mathrm{i})$. Let $f$ be a Darboux function such that (3.3) holds. Then $\mathbb{R} \backslash$ $\left(A_{0} \cup A_{1}\right)=[f \notin\{0,1\}]$ is the inverse image of an open set under $f$, so it is bilaterally $\mathfrak{c}$-dense in itself. Condition (3.2) is evident.

Corollary 3.3. Let $A_{0}, A_{1} \subset \mathbb{R}$. The following are equivalent:

(i) the sets $A_{0}$ and $A_{1}$ satisfy condition (3.2),

(ii) there is an almost continuous function $f$ such that

$$
A_{0} \subset[f=0], \quad A_{1} \subset[f=1],
$$

(iii) there is a connected function $f$ such that (3.4) holds,

(iv) there is a functionally connected function $f$ such that (3.4) holds,

(v) there is a Darboux function $f$ such that (3.4) holds.

Proof. (i) $\Rightarrow$ (ii). For $i<2$ define

$$
B_{i}=A_{i} \cup\left\{x \in \mathbb{R}: \operatorname{card}\left([x, t] \backslash A_{i}\right)<\mathfrak{c} \text { for some } t \neq x\right\} .
$$

Notice that $\mathbb{R} \backslash\left(B_{0} \cup B_{1}\right)=\left(\mathbb{R} \backslash\left(A_{0} \cup A_{1}\right)\right)^{\star}$.

Indeed, if $x \notin\left(\mathbb{R} \backslash\left(A_{0} \cup A_{1}\right)\right)^{\star}$, then $\operatorname{card}\left([x, t] \backslash\left(A_{0} \cup A_{1}\right)\right)<\mathfrak{c}$ for some $t \neq x$, and by (3.2), either $x \in B_{0}$ or $x \in B_{1}$. On the other hand, if $x \in\left(\mathbb{R} \backslash\left(A_{0} \cup A_{1}\right)\right)^{\star}$, then $\operatorname{card}\left([x, t] \backslash\left(A_{0} \cup A_{1}\right)\right)=\mathfrak{c}$ for each $t \neq x$, and consequently, $x \notin B_{0} \cup B_{1}$. It follows that $\mathbb{R} \backslash\left(B_{0} \cup B_{1}\right)=\left(\mathbb{R} \backslash\left(B_{0} \cup B_{1}\right)\right)^{\star}$.

Now assume $\operatorname{card}\left([\alpha, \beta] \backslash\left(B_{0} \cup B_{1}\right)\right)<\mathfrak{c}$ for some $\beta \neq \alpha$. Since

$\operatorname{card}\left(\left(B_{0} \cup B_{1}\right) \backslash\left(A_{0} \cup A_{1}\right)\right)=\operatorname{card}\left(\left(\mathbb{R} \backslash\left(A_{0} \cup A_{1}\right)\right) \backslash\left(\mathbb{R} \backslash\left(A_{0} \cup A_{1}\right)\right)^{\star}\right)<\mathfrak{c}$, we obtain $\operatorname{card}\left([\alpha, \beta] \backslash\left(A_{0} \cup A_{1}\right)\right)<\mathfrak{c}$. So by (3.2), either $\alpha, \beta \in A_{0} \subset B_{0}$, or $\alpha, \beta \in A_{1} \subset B_{1}$.

By Proposition 3.1, we can construct an almost continuous function $f$ such that $[f=0]=B_{0} \supset A_{0}$ and $[f=1]=B_{1} \supset A_{1}$.

The implications (ii) $\Rightarrow$ (iii) $\Rightarrow$ (iv) $\Rightarrow$ (v) are immediate.

$(\mathrm{v}) \Rightarrow(\mathrm{i})$. Let $f$ be a Darboux function such that (3.4) holds. Fix $\alpha \in A_{0}$ and $\beta \in A_{1}$. Then $f(\alpha)=0$ and $f(\beta)=1$, so by Corollary 3.2,

$$
\operatorname{card}\left([\alpha, \beta] \backslash\left(A_{0} \cup A_{1}\right)\right) \geq \operatorname{card}([\alpha, \beta] \backslash([f=0] \cup[f=1]))=\mathfrak{c} .
$$

4. New separation property. We start with two auxiliary propositions.

Proposition 4.1. Let $A^{-}, A^{+} \subset \mathbb{R}$ be such that

$$
A^{-}=\left(A^{-}\right)^{\star}, \quad A^{+}=\left(A^{+}\right)^{\star},
$$


(4.2) for each $\alpha \in A^{-}$and $\beta \in A^{+}$we have $[\alpha, \beta] \backslash\left(A^{-} \cup A^{+}\right) \neq \emptyset$.

Assume that a function $f$ satisfies the following conditions:

$$
A^{-}=[f<0], \quad A^{+}=[f>0],
$$

(4.4) $\quad f \cap K \neq \emptyset$ whenever $K \subset \mathbb{R}^{2}$ is closed and

$$
\text { card } \operatorname{dom}\left(K \cap\left(\left[A^{-} \times(-\infty, 0)\right] \cup\left[A^{+} \times(0, \infty)\right]\right)\right)=\mathfrak{c} .
$$

Then $f$ is a Darboux function. If moreover

for each closed set $F \subset A^{-} \cup A^{+}, \alpha \in A^{-} \cap F$, and $\beta \in A^{+} \cap F$, there are $\alpha^{\prime} \in A^{-} \cap[\alpha, \beta]$ and $\beta^{\prime} \in A^{+}$such that $F \cap\left[\alpha^{\prime}, \beta^{\prime}\right]=\emptyset$,

then $f$ is connected. If moreover

(4.6) for each closed set $F \subset A^{-} \cup A^{+}, \alpha \in A^{-} \cap F$, and $\beta \in A^{+} \cap F$, there are $\alpha^{\prime} \in A^{-} \cap[\alpha, \beta]$ and $\beta^{\prime} \in A^{+}$such that $F \cap\left[\alpha^{\prime}, \beta^{\prime}\right]=\emptyset$ and $\left(\beta^{\prime}-\alpha^{\prime}\right)(\beta-\alpha)>0$,

then $f$ is almost continuous.

Proof. First observe that

$$
\text { if } a \in A^{+} \text {and } b \neq a \text {, then } f[[a, b]] \supset(0, \infty) \text {. }
$$

Indeed, by (4.1) and (4.4), for each $y>0$ we have $f \cap[[a, b] \times\{y\}] \neq \emptyset$. Similarly we can show that if $a \in A^{-}$and $b \neq a$, then $f[[a, b]] \supset(-\infty, 0)$.

To prove that $f$ is a Darboux function fix $a, b \in \mathbb{R}$ and $y \in(f(a), f(b))$. If $y>0$, then by (4.3), we obtain $b \in A^{+}$. So by (4.7), $f(x)=y$ for some $x \in[a, b]$. Similarly we proceed if $y<0$. Finally if $y=0$, then by (4.3) and (4.2), $f(x)=0$ for some $x \in[a, b]$.

The rest of the proof of the proposition consists of several auxiliary claims. The end of the proof of each claim will be marked with $\triangleleft$.

Claim 1. Let $G$ denote the set of all $x \in \mathbb{R}$ such that $f$ is almost continuous in some neighborhood of $x$. If $a<b$ and $[a, b] \subset G$, then $f\lceil[a, b]$ is almost continuous.

For each $x \in[a, b]$ there is a $\delta_{x}>0$ such that $f \uparrow\left(x-\delta_{x}, x+\delta_{x}\right)$ is almost continuous. Now, the relation $[a, b] \subset \bigcup_{x \in[a, b]}\left(x-\delta_{x}, x+\delta_{x}\right)$ and the compactness of $[a, b]$ imply that $[a, b] \subset \bigcup_{i=0}^{p}\left(x_{i}-\delta_{x_{i}}, x_{i}+\delta_{x_{i}}\right)$ for some $x_{0}, \ldots, x_{p} \in[a, b]$. Hence we can find $a=t_{0}<\ldots<t_{l}=b$ such that $f \uparrow\left[t_{j-1}, t_{j}\right]$ is almost continuous for each $j$. Thus $f \uparrow[a, b]$ is almost continuous as well. $\triangleleft$

Claim 2. If $a<b$ and $(a, b) \subset G$, then $f\lceil[a, b]$ is almost continuous.

Using Claim 1 we can easily show that $f \uparrow(a, b)$ is almost continuous. (See also [4] or [5, Corollary 2.2].) We have already proved that $f$ is a Darboux function. Hence $\langle a, f(a)\rangle,\langle b, f(b)\rangle \in \operatorname{cl}(f \uparrow(a, b))$. So, $f \uparrow[a, b]$ is almost continuous. (Cf. [5, Theorem 2.4] or [3].) $\triangleleft$ 
Claim 3. Let $V \subset \mathbb{R}^{2}$ be an arbitrary open set containing $f$, and let $\mathrm{R}(x, \delta) \subset V$. For each $t \in(x-\delta, x+\delta)$, if $f(x) f(t) \geq 0$ and $f(t) \neq 0$, then there is a continuous function $h:[x, t] \rightarrow \mathbb{R}$ contained in $V$ such that $h=f$ on $\{x, t\}$.

Assume that $x<t$ and $f(t)>0$. (The other cases are similar.) Since the set $\mathbb{R}^{2} \backslash V$ is closed and $f \subset V$, condition (4.4) implies

$$
\operatorname{card}\left\{z \in A^{+}:\{z\} \times(0, \infty) \not \subset V\right\}=\operatorname{card} \operatorname{dom}\left(\left[A^{+} \times(0, \infty)\right] \backslash V\right)<\mathfrak{c} .
$$

Let $\tau>0$ be such that $x<t-\tau$ and $\mathrm{R}(t, \tau) \subset V$. Then by (4.1), there is a $z \in(t-\tau, t)$ such that $\{z\} \times(0, \infty) \subset V$. Using the compactness of the set $\{z\} \times[f(x)+\delta, f(t)] \subset V$, we can find an $\eta>0$ such that $z+\eta<t$ and $(z-\eta, z+\eta) \times[f(x)+\delta, f(t)] \subset V$. Define $h:[x, t] \rightarrow \mathbb{R}$ by connecting the following pairs of points by straight line segments: $\langle x, f(x)\rangle$ with $\langle z, f(x)+\delta\rangle$, $\langle z, f(x)+\delta\rangle$ with $\langle z+\eta, f(t)\rangle$, and $\langle z+\eta, f(t)\rangle$ with $\langle t, f(t)\rangle . \triangleleft$

Claim 4. Let $V \subset \mathbb{R}^{2}$ be an arbitrary open set containing $f$, and let $\mathrm{R}(x, \delta) \subset V$, where $x \notin A^{-}$. For each $t \in(x, x+\delta)$, if $t \in \operatorname{cl}\left(A^{+} \cap(-\infty, t)\right)$ and $\langle t, 0\rangle \in V$, then there is a continuous function $h:[x, t] \rightarrow \mathbb{R}$ contained in $V$ such that $h(x)=f(x)$ and $h(t)=0\left({ }^{1}\right)$.

Let $\tau>0$ be such that $x<t-\tau$ and $(t-\tau, t+\tau) \times(-\tau, \tau) \subset V$. By (4.7), there is a $z \in(t-\tau, t)$ such that $f(z)=\tau$. So by Claim 3 , there is a continuous function $h_{0}:[x, z] \rightarrow \mathbb{R}$ contained in $V$ such that $h=f$ on $\{x, z\}$. Extend $h_{0}$ to $h:[x, t] \rightarrow \mathbb{R}$ by connecting $\langle z, f(z)\rangle$ with $\langle t, 0\rangle$ by a straight line segment. $\triangleleft$

Claim 5. Let $P=\mathbb{R} \backslash G$. Then $P \cap\left(A^{-} \cup A^{+}\right)$is dense in $P$.

By way of contradiction suppose that there is a closed interval $J$ such that $P \cap \operatorname{Int} J \neq \emptyset=P \cap\left(A^{-} \cup A^{+}\right) \cap J$. Then by Claim 2, $f\lceil$ cl I is almost continuous for each connected component $I$ of $J \backslash P$. On the other hand by (4.3), $P \cap J \subset[f=0]$. Using [5, Lemma 6.3] we find that $f\lceil J$ is almost continuous. It follows that $\emptyset \neq P \cap$ Int $J \subset$ Int $J \subset G$, an impossibility. $\triangleleft$

Claim 6. Both $P \cap A^{-}$and $P \cap A^{+}$are dense in $P$.

By way of contradiction suppose that $P \cap(a, b) \neq \emptyset=P \cap[a, b] \cap A^{-}$for some $a<b$. (The other case is similar.) We will show that $f\lceil[a, b]$ is almost continuous, which contradicts $P \cap(a, b) \neq \emptyset$.

Take an arbitrary open set $V \supset f\lceil[a, b]$. Let $S$ be the set of all $s \in[a, b]$ for which there is a continuous function $h:[s, b] \rightarrow \mathbb{R}$ contained in $V$ such that $h=f$ on $\{s, b\}$. Define $x_{0}=\inf S$. First we will prove that $x_{0} \in S$.

Indeed, suppose that this is not the case. Then $x_{0}<b$. Let $\delta>0$ be such that $\mathrm{R}\left(x_{0}, \delta\right) \subset V$. Fix an $x \in S \cap\left(x_{0}, x_{0}+\delta\right)$. Let $h_{0}$ correspond to $x \in S$.

$\left({ }^{1}\right)$ Notice that we can allow $t \in(x-\delta, x)$ provided that $t \in \operatorname{cl}\left(A^{+} \cap(t, \infty)\right)$. We can also prove analogous claims in which we swap the symbols $A^{-}$and $A^{+}$. 
Set $x_{1}=\sup (P \cap(-\infty, x))$. By Claim $2, f\left\lceil\left[x_{1}, x\right]\right.$ is almost continuous, so there is a continuous function $h_{1}:\left[x_{1}, x\right] \rightarrow \mathbb{R}$ contained in $V$ such that $f=h_{1}$ on $\left\{x_{1}, x\right\}$. (See [5, Lemma 6.2].) Then the function $h=h_{0} \cup h_{1}$ proves $x_{1} \in S$, whence $x_{1}>x_{0}$. By Claim 3 or 4 , there is a continuous function $h_{2}:\left[x_{0}, x_{1}\right] \rightarrow \mathbb{R}$ contained in $V$ such that $h_{2}=f$ on $\left\{x_{0}, x_{1}\right\}$. Clearly the function $h=h_{0} \cup h_{1} \cup h_{2}$ proves $x_{0} \in S$, contrary to assumption.

Now suppose that $x_{0}>a$. Let $\delta>0$ be such that $a<x_{0}-\delta$ and $\mathrm{R}\left(x_{0}, \delta\right) \subset V$. Since $f$ is a Darboux function, we can choose an $x \in\left(x_{0}-\right.$ $\left.\delta, x_{0}\right)$ such that $\left|f(x)-f\left(x_{0}\right)\right|<\delta$. Let $h_{0}$ correspond to $x_{0} \in S$. Extend $h_{0}$ to $h:[x, b] \rightarrow \mathbb{R}$ by connecting $\langle x, f(x)\rangle$ with $\left\langle x_{0}, f\left(x_{0}\right)\right\rangle$ by a straight line segment. Clearly this function proves $x \in S$. But $x<x_{0}=\inf S$, an impossibility.

We proved that $a \in S$. So, there is a continuous function $h:[a, b] \rightarrow \mathbb{R}$ contained in $V$. Since $V$ was an arbitrary open set containing $f \uparrow[a, b]$, it follows that $f\lceil[a, b]$ is almost continuous and $(a, b) \subset G$. But $P \cap(a, b) \neq \emptyset$, a contradiction. $\triangleleft$

Claim 7. $P \cap[f=0]$ is nowhere dense in $P$.

By way of contradiction suppose that the set $P \cap[f=0] \cap(a, b)$ is dense in $P \cap(a, b) \neq \emptyset$ for some $a<b$. We will show that $f \uparrow[a, b]$ is almost continuous, which contradicts $P \cap(a, b) \neq \emptyset$.

Take an arbitrary open set $V \supset f \uparrow[a, b]$. Let $S$ be the set of all $s \in[a, b]$ for which there is a continuous function $h:[s, b] \rightarrow \mathbb{R}$ contained in $V$ such that $h=f$ on $\{s, b\}$. Define $x_{0}=\inf S$. First we will prove that $x_{0} \in S$.

Indeed, suppose that this is not the case. Then $x_{0}<b$. Let $\delta>0$ be such that $\mathrm{R}\left(x_{0}, \delta\right) \subset V$. Fix an $x \in S \cap\left(x_{0}, x_{0}+\delta\right)$. Let $h_{0}$ correspond to $x \in S$. Set $x_{1}=\sup (P \cap(-\infty, x))$. By Claim 2, there is a continuous function $h_{1}:\left[x_{1}, x\right] \rightarrow \mathbb{R}$ contained in $V$ such that $f=h_{1}$ on $\left\{x_{1}, x\right\}$. Thus $x_{1} \in S$ and $x_{1}>x_{0}$. Let $\tau>0$ be such that $x_{0}<x_{1}-\tau$ and $\mathrm{R}\left(x_{1}, \tau\right) \subset V$. Pick an $x_{2} \in P \cap\left(x_{1}-\tau, x_{1}\right) \cap[f=0]$. Let $\eta>0$ be such that $\mathrm{R}\left(x_{2}, \eta\right) \subset V$. Choose an $x_{3} \in P \cap\left(x_{2}-\eta, x_{2}+\eta\right) \cap\left(x_{1}-\tau, x_{1}\right)$ which is a bilateral limit point of $P$. By Claim 4 , there are continuous functions $h_{2}:\left[x_{0}, x_{3}\right] \rightarrow \mathbb{R}$ and $h_{3}:\left[x_{3}, x_{1}\right] \rightarrow \mathbb{R}$ contained in $V$ such that $h_{2}\left(x_{0}\right)=f\left(x_{0}\right), h_{2}\left(x_{3}\right)=h_{3}\left(x_{3}\right)=0$, and $h_{3}\left(x_{1}\right)=f\left(x_{1}\right)$. One can easily see that the function $h=h_{0} \cup \ldots \cup h_{3}$ proves $x_{0} \in S$, which contradicts our supposition.

Proceeding as in the proof of Claim 6 , we can show that $x_{0}=a$. So, there is a continuous function $h:[a, b] \rightarrow \mathbb{R}$ contained in $V$. Since $V$ was an arbitrary open set containing $f\lceil[a, b]$, it follows that $f \uparrow[a, b]$ is almost continuous and $(a, b) \subset G$. But $P \cap(a, b) \neq \emptyset$, an impossibility. $\triangleleft$

Claim 8. If condition (4.5) holds, then $f$ is connected. 
Take an open set $V \supset f$. We will show that for all $a<b$ there is a connected set $h \subset \mathbb{R}^{2}$ contained in $V$ such that $\langle a, f(a)\rangle,\langle b, f(b)\rangle \in h$. Let $a<b$ be arbitrary and let $S$ be the set of all $s \in[a, b]$ for which there is a connected set $h \subset \mathbb{R}^{2}$ contained in $V$ such that $\langle s, f(s)\rangle,\langle b, f(b)\rangle \in h$. Define $x_{0}=\inf S$. First we will prove that $x_{0} \in S$.

Indeed, suppose that this is not the case. Then $x_{0}<b$. Let $\delta>0$ be such that $\mathrm{R}\left(x_{0}, \delta\right) \subset V$. Fix an $x \in S \cap\left(x_{0}, x_{0}+\delta\right)$. Let $h_{0}$ correspond to $x \in S$. Set $x_{1}=\sup (P \cap(-\infty, x))$. By Claim 2 , there is a continuous function $h_{1}:\left[x_{1}, x\right] \rightarrow \mathbb{R}$ contained in $V$ such that $f=h_{1}$ on $\left\{x_{1}, x\right\}$. Then the set $h=h_{0} \cup h_{1}$ proves $x_{1} \in S$, whence $x_{1}>x_{0}$. If $f\left(x_{0}\right) f\left(x_{1}\right) \geq 0$, then by Claim 3 or 4 , there is a continuous function $h_{2}:\left[x_{0}, x_{1}\right] \rightarrow \mathbb{R}$ contained in $V$ such that $f=h_{2}$ on $\left\{x_{0}, x_{1}\right\}$. (See also Claim 6.) Then the set $h=h_{0} \cup h_{1} \cup h_{2}$ proves $x_{0} \in S$, contrary to assumption.

Otherwise let $\tau>0$ be such that $x_{0}<x_{1}-\tau$ and $\mathrm{R}\left(x_{1}, \tau\right) \subset V$. Use Claims 6 and 7 to find $x_{2}, x_{3} \in\left(x_{1}-\tau, x_{1}\right)$ such that $f\left(x_{0}\right) f\left(x_{2}\right)>0$, $f\left(x_{3}\right) f\left(x_{1}\right)>0, x_{2}<x_{3}$, and $P \cap\left[x_{2}, x_{3}\right] \cap[f=0]=\emptyset$. By (4.5), there are $x_{4}, x_{5} \in\left[x_{2}, x_{3}\right]$ such that $f\left(x_{4}\right) f\left(x_{5}\right)<0$ and $P \cap\left[x_{4}, x_{5}\right]=\emptyset$. Clearly we may assume that $f\left(x_{0}\right) f\left(x_{4}\right)>0$ and $f\left(x_{5}\right) f\left(x_{1}\right)>0$. By Claim 3, there are continuous functions $h_{2}:\left[x_{0}, x_{4}\right] \rightarrow \mathbb{R}$ and $h_{3}:\left[x_{5}, x_{1}\right] \rightarrow \mathbb{R}$ contained in $V$ such that $h_{2}=f$ on $\left\{x_{0}, x_{4}\right\}$ and $h_{3}=f$ on $\left\{x_{5}, x_{1}\right\}$. By Claim 1 , there is a continuous function $h_{4}:\left[x_{4}, x_{5}\right] \rightarrow \mathbb{R}$ contained in $V$ such that $f=h_{4}$ on $\left\{x_{4}, x_{5}\right\}$. One can easily see that the set $h=h_{0} \cup \ldots \cup h_{4}$ proves $x_{0} \in S$, which contradicts our supposition. Notice that $h$ need not be a function.

Proceeding as in the proof of Claim 6 we can show that $x_{0}=a$. Consequently, $\langle a, f(a)\rangle$ and $\langle b, f(b)\rangle$ belong to the same connected component of $V$. Since the open set $V \supset f$ was arbitrary, we conclude that the function $f$ is connected. $\triangleleft$

Claim 9. If condition (4.6) holds, then $f$ is almost continuous.

Take an open set $V \supset f$. Let $a<b$ be arbitrary and let $S$ be the set of all $s \in[a, b]$ for which there is a continuous function $h:[s, b] \rightarrow \mathbb{R}$ contained in $V$ such that $h=f$ on $\{s, b\}$. Define $x_{0}=\inf S$.

The rest of the proof is a repetition of the argument used in Claim 8 . The only difference is that in the last-but-one paragraph we moreover require that $x_{4}<x_{5}$. Then the set $h=h_{0} \cup \ldots \cup h_{4}$ constructed in that paragraph is a function, which proves $x_{0} \in S$.

REMARK. It can be readily observed (by the above proof) that conditions (4.5) and (4.6) in Proposition 4.1 can be weakened. Namely, it suffices to require that their assertions hold for nowhere dense, perfect sets $F \subset A^{-} \cup A^{+}$such that both $F \cap A^{-}$and $F \cap A^{+}$are dense in $F$. 
Proposition 4.2. Let $A^{-}, A^{+} \subset \mathbb{R}$ be disjoint. Then there is a function $f$ which satisfies conditions (4.3) and (4.4).

Proof. Let $\left\{K_{\xi}: \xi<\mathfrak{c}\right\}$ be an enumeration of all closed sets $K \subset \mathbb{R}^{2}$ such that card $\operatorname{dom}\left(K \cap\left(\left[A^{-} \times(-\infty, 0)\right] \cup\left[A^{+} \times(0, \infty)\right]\right)\right)=\mathfrak{c}$. For each $\xi<\mathfrak{c}$ choose a point $\left\langle x_{\xi}, y_{\xi}\right\rangle \in K_{\xi} \cap\left(\left[A^{-} \times(-\infty, 0)\right] \cup\left[A^{+} \times(0, \infty)\right]\right)$ such that $x_{\xi} \notin\left\{x_{\zeta}: \zeta<\xi\right\}$. Define

$$
f(x)= \begin{cases}y_{\xi} & \text { if } x=x_{\xi}, \xi<\mathfrak{c} \\ -1 & \text { if } x \in A^{-} \backslash\left\{x_{\xi}: \xi<\mathfrak{c}\right\} \\ 1 & \text { if } x \in A^{+} \backslash\left\{x_{\xi}: \xi<\mathfrak{c}\right\} \\ 0 & \text { otherwise }\end{cases}
$$

Clearly the function $f$ satisfies (4.3) and (4.4).

Theorem 4.3. Let $A^{-}, A^{+} \subset \mathbb{R}$. The following are equivalent:

(i) the sets $A^{-}$and $A^{+}$satisfy conditions (4.1) and (4.2),

(ii) there is a Darboux function $f$ such that (4.3) holds.

Proof. (i) $\Rightarrow$ (ii). Use Proposition 4.2 to construct a function $f$ which satisfies (4.3) and (4.4). Then by Proposition 4.1, $f$ is a Darboux function.

The implication (ii) $\Rightarrow(\mathrm{i})$ is evident.

Theorem 4.4. Let $A^{-}, A^{+} \subset \mathbb{R}$. The following are equivalent:

(i) the sets $A^{-}$and $A^{+}$satisfy conditions (4.1), (4.2), and (4.5),

(ii) there is a connected function $f$ such that (4.3) holds,

(iii) there is a functionally connected function $f$ such that (4.3) holds.

Proof. (i) $\Rightarrow$ (ii). Use Proposition 4.2 to construct a function $f$ which satisfies (4.3) and (4.4). Then by Proposition 4.1, $f$ is connected.

The implication (ii) $\Rightarrow$ (iii) is evident.

(iii) $\Rightarrow$ (i). Let $f$ be a functionally connected function such that (4.3) holds. Then (4.1) and (4.2) follow by Theorem 4.3. To prove (4.5) fix a closed set $F \subset A^{-} \cup A^{+}, \alpha \in A^{-} \cap F$, and $\beta \in A^{+} \cap F$. Arrange all connected components of $[\alpha, \beta] \backslash F$ in a sequence, $\left\{I_{n}: n \in N\right\}$. (We have $N=\mathbb{N} \cap(0, r)$ for some $r \in(0, \infty]$.) Define

$$
N^{-}=\left\{n \in N: I_{n} \subset[f \leq 0]\right\}, \quad N^{+}=\left\{n \in N \backslash N^{-}: I_{n} \subset[f \geq 0]\right\},
$$

and suppose that contrary to (4.5), $N=N^{-} \cup N^{+}$. For each $n$ let $g_{n}$ : $\mathbb{R} \rightarrow\left[0, n^{-1}\right]$ be a continuous function such that $I_{n}=\left[g_{n}>0\right]$. Define the function $g:[\alpha, \beta] \rightarrow \mathbb{R}$ by

$$
g(x)= \begin{cases}g_{n}(x) & \text { if } x \in I_{n}, n \in N^{-} \\ -g_{n}(x) & \text { if } x \in I_{n}, n \in N^{+} \\ 0 & \text { otherwise }\end{cases}
$$


Then $g$ is continuous, $f(\alpha)<0=g(\alpha)$, and $f(\beta)>0=g(\beta)$. But $g \cap f=\emptyset$ (recall that $F \subset A^{-} \cup A^{+}$), so $f$ is not functionally connected, contrary to assumption.

TheOREM 4.5. Let $A^{-}, A^{+} \subset \mathbb{R}$. The following are equivalent:

(i) the sets $A^{-}$and $A^{+}$satisfy conditions (4.1), (4.2), and (4.6),

(ii) there is an almost continuous function $f$ such that (4.3) holds.

Proof. (i) $\Rightarrow$ (ii). Use Proposition 4.2 to construct a function $f$ which satisfies (4.3) and (4.4). Then by Proposition 4.1, $f$ is almost continuous.

(ii) $\Rightarrow$ (i). Let $f$ be an almost continuous function such that (4.3) holds. Then (4.1) and (4.2) follow by Theorem 4.3. To prove (4.6) fix a closed set $F \subset A^{-} \cup A^{+}, \alpha \in A^{-} \cap F$, and $\beta \in A^{+} \cap F$. Assume that $\alpha<\beta$ (the other case is analogous), and arrange all connected components of $[\alpha, \beta] \backslash F$ in a sequence, $\left\{I_{n}: n \in N\right\}$. For each $n \in N$ let $I_{n}=\left(\alpha_{n}, \beta_{n}\right)$. Define $N^{-}$ and $N^{+}$by (4.8), and denote by $N^{\prime}$ the set of all $n \in N \backslash\left(N^{-} \cup N^{+}\right)$for which there is a $\gamma_{n} \in I_{n}$ such that $\left(\alpha_{n}, \gamma_{n}\right] \subset[f \geq 0]$ and $\left[\gamma_{n}, \beta_{n}\right) \subset[f \leq 0]$. Suppose that contrary to (4.6), $N=N^{-} \cup N^{+} \cup N^{\prime}$. If $n \in N^{-}$, then let $K_{n}$ be the union of the line segments connecting $\left\langle\alpha_{n}, 0\right\rangle$ with $\left\langle\left(\alpha_{n}+\beta_{n}\right) / 2, n^{-1}\right\rangle$ and $\left\langle\left(\alpha_{n}+\beta_{n}\right) / 2, n^{-1}\right\rangle$ with $\left\langle\beta_{n}, 0\right\rangle$. If $n \in N^{+}$, then let $K_{n}$ be the union of the segments connecting $\left\langle\alpha_{n}, 0\right\rangle$ with $\left\langle\left(\alpha_{n}+\beta_{n}\right) / 2,-n^{-1}\right\rangle$ and $\left\langle\left(\alpha_{n}+\beta_{n}\right) / 2\right.$, $\left.-n^{-1}\right\rangle$ with $\left\langle\beta_{n}, 0\right\rangle$. Finally if $n \in N^{\prime}$, then let $K_{n}$ be the union of the segments connecting $\left\langle\alpha_{n}, 0\right\rangle$ with $\left\langle\gamma_{n},-n^{-1}\right\rangle$ and $\left\langle\gamma_{n}, n^{-1}\right\rangle$ with $\left\langle\beta_{n}, 0\right\rangle$. Set

$$
K=[\{\alpha\} \times[0, \infty)] \cup \bigcup_{n \in N} K_{n} \cup[F \times\{0\}] \cup[\{\beta\} \times(-\infty, 0]]
$$

and $U=\mathbb{R}^{2} \backslash K$. Then $U$ is open and $U \supset f$. Suppose that there is a continuous function $g \subset U$. Observe that $g(\alpha)<0<g(\beta)$, and set

$$
x=\max (F \cap[\alpha, \beta] \cap[g \leq 0]) .
$$

Since $g(x) \leq 0$ and $x \in F$, we obtain $g(x)<0$. If $x=\alpha_{n}$ for some $n \in N$, then using the fact that $g \cap K_{n}=\emptyset$, we get $g\left(\beta_{n}\right)<0$, which contradicts the definition of $x$. Otherwise $x \in \operatorname{cl}(F \cap[g>0]$ ) (notice that $x<\beta)$, which is also impossible.

Proposition 4.6 will be helpful in proving theorems analogous to the above ones in which we do not require $f=0$ on $\mathbb{R} \backslash\left(A^{-} \cup A^{+}\right)$.

Proposition 4.6. Assume that the sets $A^{-}, A^{+} \subset \mathbb{R}$ satisfy condition (4.2) and

$$
A^{-} \subset\left(\mathbb{R} \backslash A^{+}\right)^{\star}, \quad A^{+} \subset\left(\mathbb{R} \backslash A^{-}\right)^{\star} .
$$

Then there is a Darboux function $f$ such that

$$
A^{-} \subset[f<0], \quad A^{+} \subset[f>0] .
$$


If $A^{-}$and $A^{+}$satisfy moreover (4.5), then $f$ is connected. If $A^{-}$and $A^{+}$ satisfy moreover (4.6), then $f$ is almost continuous.

Proof. Clearly we may assume that condition (4.1) fails. Let $\left\{F_{\xi}: \xi<\mathfrak{c}\right\}$ be an enumeration of all closed sets $F \subset \mathbb{R}$ such that $\operatorname{card}\left(F \backslash\left(A^{-} \cup A^{+}\right)\right)=\mathfrak{c}$. For each $\xi<\mathfrak{c}$ choose different points

$$
x_{\xi, 0}, x_{\xi, 1}, x_{\xi, 2} \in\left(F_{\xi} \backslash\left(A^{-} \cup A^{+}\right)\right)^{\star} \backslash\left\{x_{\zeta, i}: \zeta<\xi, i<3\right\} .
$$

Define

$$
B^{-}=A^{-} \cup\left\{x_{\xi, 0}: \xi<\mathfrak{c}\right\}, \quad B^{+}=A^{+} \cup\left\{x_{\xi, 1}: \xi<\mathfrak{c}\right\} .
$$

Let $\alpha \in B^{-}$and $\beta \neq \alpha$. If $\operatorname{card}\left([\alpha, \beta] \backslash\left(A^{-} \cup A^{+}\right)\right)<\mathfrak{c}$, then $\alpha \in A^{-}$ and by (4.9),

$$
\operatorname{card}\left([\alpha, \beta] \cap B^{-}\right) \geq \operatorname{card}\left([\alpha, \beta] \cap A^{-}\right)=\mathfrak{c} .
$$

In the opposite case $[\alpha, \beta]=F_{\xi}$ for some $\xi<\mathfrak{c}$, so

$$
\operatorname{card}\left([\alpha, \beta] \cap B^{-}\right) \geq \operatorname{card}\left\{x_{\zeta, 0}: \zeta<\mathfrak{c}, F_{\zeta} \subset F_{\xi}\right\}=\mathfrak{c} .
$$

Consequently, $B^{-}=\left(B^{-}\right)^{\star}$. Similarly we can show that $B^{+}=\left(B^{+}\right)^{\star}$.

Now let $\alpha \in B^{-}$and $\beta \in B^{+}$. If $x_{\xi, i} \in[\alpha, \beta]$ for some $\xi<\mathfrak{c}$ and $i<2$, then $[\alpha, \beta]=F_{\zeta}$ for some $\zeta<\mathfrak{c}$, and $x_{\zeta, 2} \in[\alpha, \beta] \backslash\left(B^{-} \cup B^{+}\right)$. In the opposite case by $(4.2),[\alpha, \beta] \backslash\left(B^{-} \cup B^{+}\right)=[\alpha, \beta] \backslash\left(A^{-} \cup A^{+}\right) \neq \emptyset$.

By Proposition 4.2, there exists a function $f$ such that $B^{-}=[f<0]$, $B^{+}=[f>0]$, and $f \cap K \neq \emptyset$ whenever $K \subset \mathbb{R}^{2}$ is closed and

$$
\operatorname{card} \operatorname{dom}\left(K \cap\left(\left[B^{-} \times(-\infty, 0)\right] \cup\left[B^{+} \times(0, \infty)\right]\right)\right)=\mathfrak{c} .
$$

Then by Proposition 4.1, $f$ is a Darboux function.

Now assume that $A^{-}$and $A^{+}$satisfy also (4.5). Fix a closed set $F \subset$ $B^{-} \cup B^{+}, \alpha \in B^{-} \cap F$, and $\beta \in B^{+} \cap F$. If $F \subset A^{-} \cup A^{+}$, then by (4.5), there exist $\alpha^{\prime} \in B^{-} \cap[\alpha, \beta]$ and $\beta^{\prime} \in B^{+}$such that $F \cap\left[\alpha^{\prime}, \beta^{\prime}\right]=\emptyset$.

Otherwise $\operatorname{card}\left([\alpha, \beta] \backslash\left(A^{-} \cup A^{+}\right)\right)=\mathfrak{c}$. Notice that

$$
\left\{x_{\xi, 2}: \xi<\mathfrak{c}\right\} \cap F \subset\left\{x_{\xi, 2}: \xi<\mathfrak{c}\right\} \cap\left(B^{-} \cup B^{+}\right)=\emptyset,
$$

and conclude that $\operatorname{card}\left(F \backslash\left(A^{-} \cup A^{+}\right)\right)<\mathfrak{c}$. Consequently, there is a connected component $I$ of $[\alpha, \beta] \backslash F$ such that $\operatorname{cl} I=F_{\xi}$ for some $\xi<\mathfrak{c}$. Then $\alpha^{\prime}=x_{\xi, 0} \in B^{-} \cap[\alpha, \beta], \beta^{\prime}=x_{\xi, 1} \in B^{+}$, and $F \cap\left[\alpha^{\prime}, \beta^{\prime}\right]=\emptyset$. So by Proposition $4.1, f$ is connected.

Finally assume that $A^{-}$and $A^{+}$satisfy (4.6). Then arguing as in the previous case, we can conclude that $f$ is almost continuous.

Theorem 4.7. Let $A^{-}, A^{+} \subset \mathbb{R}$. The following are equivalent:

(i) the sets $A^{-}$and $A^{+}$satisfy conditions (4.2) and (4.9),

(ii) there is a Darboux function $f$ such that (4.10) holds.

Proof. The implication (i) $\Rightarrow$ (ii) follows by Proposition 4.6. 
(ii) $\Rightarrow($ i). Let $f$ be a Darboux function such that (4.10) holds. Then by Theorem 4.3,

$$
A^{-} \subset[f<0]=[f<0]^{\star} \subset\left(\mathbb{R} \backslash A^{+}\right)^{\star} .
$$

Similarly we can show that $A^{+} \subset\left(\mathbb{R} \backslash A^{-}\right)^{\star}$. Condition (4.2) is evident.

The proofs of Theorems 4.8 and 4.9 mimic the argument used above.

ThEOREM 4.8. Let $A^{-}, A^{+} \subset \mathbb{R}$. The following are equivalent:

(i) the sets $A^{-}$and $A^{+}$satisfy conditions (4.2), (4.5), and (4.9),

(ii) there is a connected function $f$ such that (4.3) holds,

(iii) there is a functionally connected function $f$ such that (4.3) holds.

TheOREm 4.9. Let $A^{-}, A^{+} \subset \mathbb{R}$. The following are equivalent:

(i) the sets $A^{-}$and $A^{+}$satisfy conditions (4.2), (4.6), and (4.9),

(ii) there is an almost continuous function $f$ such that (4.3) holds.

\section{Examples}

ExAmPLE 5.1. Let $F$ be the ternary Cantor set and let $\left\{I_{n}: n \in \mathbb{N}\right\}$ be a family of connected components of $\mathbb{R} \backslash F$ such that

$$
\operatorname{cl}\left(\bigcup_{n \in \mathbb{N}} I_{n}\right) \cap \operatorname{cl}\left(\mathbb{R} \backslash \bigcup_{n \in \mathbb{N}} I_{n}\right)=F .
$$

For each $n \in \mathbb{N}$ choose an $x_{n} \in I_{n}$. Define

$$
A^{-}=\bigcup_{n \in \mathbb{N}} \operatorname{cl} I_{n} \backslash\left\{x_{n}: n \in \mathbb{N}\right\}, \quad A^{+}=\mathbb{R} \backslash \bigcup_{n \in \mathbb{N}} \operatorname{cl} I_{n} .
$$

Then $A^{-}$and $A^{+}$satisfy (4.1) and (4.2), but they fail (4.5).

ExAmple 5.2. Let $F$ be the ternary Cantor set and let $\left\{\left(a_{n}, b_{n}\right): n \in \mathbb{N}\right\}$ be the family of all connected components of $[0,1] \backslash F$. Define

$$
A^{-}=\bigcup_{n \in \mathbb{N}}\left[a_{n},\left(a_{n}+b_{n}\right) / 2\right), \quad A^{+}=\bigcup_{n \in \mathbb{N}}\left(\left(a_{n}+b_{n}\right) / 2, b_{n}\right] \cup F^{\star} \cup(\mathbb{R} \backslash(0,1)) \text {. }
$$

Then $A^{-}$and $A^{+}$satisfy (4.1), (4.2), and (4.5), but they fail (4.6).

\section{References}

[1] J. G. Ceder and T. L. Pearson, Insertion of open functions, Duke Math. J. 35 (1968), 277-288.

[2] R. Gibson and T. Natkaniec, Darboux like functions, Real Anal. Exchange 22 (1996/97), 492-533.

[3] J. M. Jastrzębski, J. M. Jędrzejewski, and T. Natkaniec, On some subclasses of Darboux functions, Fund. Math. 138 (1991), 165-173.

[4] K. R. Kellum, Sums and limits of almost continuous functions, Colloq. Math. 31 (1974), 125-128. 
[5] T. Natkaniec, Almost continuity, Real Anal. Exchange 17 (1991-92), 462-520.

[6] J. Stallings, Fixed point theorems for connectivity maps, Fund. Math. 47 (1959), 249-263.

Department of Mathematics

Bydgoszcz Academy

Pl. Weyssenhoffa 11

85-072 Bydgoszcz, Poland

E-mail: AMal@ab-byd.edu.pl

Received 3 December 2001;

in revised form 9 August 2002 